\title{
Analytical optimization of spread and change of exponential decay of generalized Wannier functions in one dimension
}

\author{
Denis R. Nacbar ${ }^{1}$ and Alexys Bruno-Alfonso ${ }^{2}$ \\ ${ }^{1}$ Programa de Pós-Graduação em Ciência e Tecnologia de Materiais, Unesp, 17033-360 Bauru, São Paulo, Brazil \\ ${ }_{2}^{2}$ Departamento de Matemática, Faculdade de Ciências, Unesp, 17033-360 Bauru, São Paulo, Brazil \\ (Received 7 November 2011; revised manuscript received 22 March 2012; published 14 May 2012)
}

\begin{abstract}
Generalized Wannier functions of a couple of bands in a one-dimensional crystal are investigated. A lower bound for the global minimum of the total spread is obtained. Assumption of such a value being the minimum leads to a first-order differential equation for the transformation matrix. Simple analytical solutions leading to real generalized Wannier functions are presented for consecutive bands in a crystal with inversion symmetry. Results are displayed for a particle in a diatomic Kronig-Penney potential. For the lowest couple of bands, calculated single-band Wannier functions resemble orbitals of a diatomic molecule, whereas generalized Wannier functions seem like orthogonalized atomic orbitals. The latter functions are neither symmetric nor antisymmetric and display increased exponential decay. For the next two pairs of bands, Wannier functions retain their centers and symmetries, and exponential decay does not increase. Results are also shown to be in agreement with solutions of the eigenvalue problem of the band-projected position operator.
\end{abstract}

DOI: 10.1103/PhysRevB.85.195127

PACS number(s): 71.20.-b, 71.15.Dx, 31.15.aq

\section{INTRODUCTION}

During the centennial year of Gregory Hugh Wannier, nearly a hundred publications have demonstrated that functions named after him are key computational tools in several research areas, such as insulators, ${ }^{1}$ magnetism, ${ }^{2,3}$ metals, ${ }^{4}$ photonics, ${ }^{5}$ polymers,${ }^{6}$ semiconductors, ${ }^{7,8}$ superconductivity, ${ }^{9}$ and electronic transport. ${ }^{10}$ of course, applications have fueled basic research where Wannier functions (WFs) are the subject matter, ${ }^{11}$ and textbooks devote some attention to these functions. ${ }^{12-16}$ Since their introduction in $1937,{ }^{17}$ the properties of WFs have challenged the solid-state physics community. ${ }^{18-20}$ This is mainly because of their lack of uniqueness, which is a feature inherited from Bloch functions (BFs). In fact, electronic BFs are common eigenfunctions of the Hamiltonian and translation operators that are conveniently chosen as periodic functions in the reciprocal space. Hence, taking normalization into account, the complex argument of BFs is determined up to a position-independent shift that should be periodic in the wave vector. Moreover, due to its periodicity, the Bloch function of each band may be represented by a Fourier series where coefficients are the Wannier functions of the band.

Contrasting the extended nature of BFs, Wannier functions are localized in real space and their nonuniqueness opens the possibility of optimizing the localization. The case of an electron in a one-dimensional (1D) crystal with inversion symmetry was investigated by Kohn in his outstanding paper of $1959 .{ }^{21}$ He proved that the phase of the BFs may be chosen in order to produce real, symmetric or antisymmetric, and exponentially localized WFs. The existence of an additional localization in the form of a power law was demonstrated in 2001 by He and Vanderbilt. ${ }^{22}$ This was extended in 2007 to deal with maximally localized WFs of the noncentrosymmetric case. ${ }^{23}$ In the latter work, localization is measured by the variance, a criterion that was successfully used in 1997 by Marzari and Vanderbilt, ${ }^{24}$ to calculate WFs of three-dimensional crystals. Their approach leads with a generalization of WFs, in the sense established a few years after Kohn's paper by Blount, ${ }^{25}$ des Cloizeaux, ${ }^{26}$ and Eilenberger, ${ }^{27}$ and became the basis of modern computational packages, like WANNIER $90,{ }^{28}$ devoted to electronic-structure calculations. Moreover, the $1 \mathrm{D}$ version of those generalized functions has been investigated by using iterative and parallel-transport approaches. ${ }^{24,29}$

The aim of this work is to speed advances in the study of generalized Wannier functions (GWFs), by performing the analytical optimization of their total quadratic spread in the case of two consecutive bands of a 1D crystal with inversion symmetry. Several questions about the obtained GWFs are addressed, such as the possibility of being real, whether they may present increased exponential localization when compared with WFs of each band, how the inversion symmetry manifests on their shape, and what is their relation with atomic orbitals. In Sec. II, we review the construction of WFs and maximally localized GWFs. The problem is analytically solved in Sec. III, and numerical results and discussions for a diatomic Kronig-Penney model are given in Sec. IV. Final considerations are presented in Sec. V.

\section{BASIC CONCEPTS AND EQUATIONS}

Let us first recall basic ideas on Wannier functions of a 1D crystal. We denote a Bloch function of band index $j$ and wave number $k$ as $\psi_{j, k}(x)$. This family of functions satisfies the Schrödinger equation $\hat{H} \psi_{j, k}(x)=E_{j}(k) \psi_{j, k}(x)$, the Bloch condition $\psi_{j, k}(x+a)=e^{i k a} \psi_{j, k}(x)$, where $a$ is the lattice period, and the orthonormalization condition $\left\langle\psi_{j, k}^{*} \psi_{j^{\prime}, k^{\prime}}\right\rangle=$ $2 \pi a^{-1} \delta_{j, j^{\prime}} \delta\left(k-k^{\prime}\right)$, for any $k$ and $k^{\prime}$ in the first Brillouin zone, where brackets refer to integration over the whole $x$ axis. Moreover, since $\psi_{j, k+2 \pi / a}(x)=\psi_{j, k}(x)$, BFs are given by the Fourier series

$$
\psi_{j, k}(x)=\sum_{n} w_{j, n}(x) e^{i k n a},
$$

where the coefficient $w_{j, n}(x)$ is the Wannier function of the $j$ th band and $n$th cell. This set of functions is orthonormal over the $x$ axis and satisfies $w_{j, n}(x)=w_{j}(x-n a)$ with 
$w_{j}(x)=\overline{\psi_{j, k}(x)}$, where the line above the expression indicates the mean value over a Brillouin zone. We do not lose generality by assuming that Wannier functions of each band are real; ${ }^{23}$ i.e., BFs satisfy $\psi_{j,-k}(x)=\psi_{j, k}^{*}(x)$. More importantly, we suppose such WFs present maximal localization, i.e., minimal variance. $^{24}$

To deal with Bloch functions of a group of $J$ bands, we consider a row $J$-vector $\Psi_{k}$ whose $j$ th component is $\psi_{j, k}$. The orthonormalization of the BFs reads $\left\langle\Psi_{k}^{\dagger} \Psi_{k^{\prime}}\right\rangle=2 \pi a^{-1} \delta(k-$ $\left.k^{\prime}\right) \mathbb{I}$. Moreover, we introduce the $J$-vector Wannier function as $W=\overline{\Psi_{k}}$. Its normalization is written as $\left\langle W^{\dagger} W\right\rangle=\mathbb{I}$.

The centers of the WFs are the diagonal elements of the following matrix:

$$
\left\langle W^{\dagger} x W\right\rangle=\left\langle x \overline{\Psi_{k}^{\dagger}} \overline{\Psi_{k^{\prime}}}\right\rangle=\overline{\mathbb{X}(k)},
$$

with

$$
\mathbb{X}(k)=i \int_{0}^{a} u_{k}^{\dagger} \dot{u}_{k} d x
$$

being the Berry connection. ${ }^{1}$ Here $u_{k}=\exp (-i k x) \Psi_{k}$ is the periodic part of $\Psi_{k}$ and a dot over a variable refers to differentiation with respect to $k$. More clearly, the center of $w_{j}(x)$ is $x_{j}=\left\langle W^{\dagger} x W\right\rangle_{j, j}=\overline{X_{j, j}(k)}$. It may be shown that $\mathbb{X}(k)$ is Hermitian and satisfies $\mathbb{X}(k+2 \pi / a)=\mathbb{X}(k)$.

To find the variance of WFs we define the matrix

$$
\left\langle W^{\dagger} x^{2} W\right\rangle=\left\langle x^{2} \overline{\Psi_{k}^{\dagger}} \overline{\Psi_{k^{\prime}}}\right\rangle=\overline{\mathbb{Y}(k)},
$$

where

$$
\mathbb{Y}(k)=\int_{0}^{a} \dot{u}_{k}^{\dagger} \dot{u}_{k} d x
$$

is a Hermitian matrix satisfying $\mathbb{Y}(k+2 \pi / a)=\mathbb{Y}(k)$. Hence, the variance of $w_{j}(x)$ is given by

$$
\sigma_{j}^{2}=\left\langle W^{\dagger} x^{2} W\right\rangle_{j, j}-x_{j}^{2}=\overline{Y_{j, j}(k)}-\left(\overline{X_{j, j}(k)}\right)^{2} .
$$

One may construct a set of $J$ multiband Bloch functions $\tilde{\psi}_{j, k}(x)$ by performing linear combinations of the $J$ pure functions $\psi_{j, k}(x)$. To do so, we introduce a $J \times J$ matrix $\mathbb{U}(k)$ such that ${ }^{24}$

$$
\tilde{\psi}_{j, k}(x)=\sum_{j^{\prime}=1}^{J} \psi_{j^{\prime}, k}(x) U_{j^{\prime}, j}(k)
$$

applies for $j=1, \ldots, J$. These are also called quasi-Bloch functions, since they satisfy the Bloch condition but are not eigenfunctions of the Hamiltonian operator.

By introducing the row $J$-vector $\tilde{\Psi}_{k}$ with $j$ component $\tilde{\psi}_{j, k}$, we are able to rewrite Eq. (7) as $\tilde{\Psi}_{k}=\Psi_{k} \mathbb{U}(k)$. Since $\left\langle\tilde{\Psi}_{k}^{\dagger} \tilde{\Psi}_{k^{\prime}}\right\rangle=2 \pi a^{-1} \delta\left(k-k^{\prime}\right) \mathbb{U}^{\dagger}(k) \mathbb{U}(k)$, Bloch functions remain orthonormalized provided $\mathbb{U}(k)$ is unitary, i.e., $\mathbb{U}^{\dagger}(k) \mathbb{U}(k)=\mathbb{I}$. Moreover, the periodicity of $\tilde{\Psi}_{k}$ in $k$, with period $2 \pi / a$, leads to $\mathbb{U}(k+2 \pi / a)=\mathbb{U}(k)$. Therefore, GWFs are given by $\tilde{W}=\bar{\Psi}_{k}$ and satisfy the orthonormalization condition $\left\langle\tilde{W}^{\dagger} \tilde{W}\right\rangle=\mathbb{I}$.

The center of the $j$ th generalized Wannier function is $\tilde{x}_{j}=$ $\left\langle\tilde{W}^{\dagger} x \tilde{W}\right\rangle_{j, j}=\overline{\tilde{X}_{j, j}(k)}$, where

$$
\tilde{\mathbb{X}}(k)=\mathbb{U}^{\dagger}(k) \mathbb{X}(k) \mathbb{U}(k)+i \mathbb{U}^{\dagger}(k) \dot{U}(k)
$$

is a Hermitian matrix. Moreover, the variance of $\tilde{w}_{j}(x)$ is given by

$$
\tilde{\sigma}_{j}^{2}=\left\langle\tilde{W}^{\dagger} x^{2} \tilde{W}\right\rangle_{j, j}-\tilde{x}_{j}^{2}=\overline{\tilde{Y}_{j, j}(k)}-\left(\overline{\tilde{X}_{j, j}(k)}\right)^{2},
$$

where

$$
\begin{aligned}
\tilde{\mathbb{Y}}(k)= & \mathbb{U}^{\dagger}(k) \mathbb{Y}(k) \mathbb{U}(k)+\dot{U}^{\dagger}(k) \dot{\mathbb{U}}(k) \\
& +i\left[\mathbb{U}^{\dagger}(k) \mathbb{X}(k) \dot{U}(k)-\dot{U}^{\dagger}(k) \mathbb{X}(k) \mathbb{U}(k)\right] \\
= & \mathbb{U}^{\dagger}(k)\left[\mathbb{Y}(k)-\mathbb{X}^{2}(k)\right] \mathbb{U}(k)+\tilde{\mathbb{X}}^{\dagger}(k) \tilde{\mathbb{X}}(k) .
\end{aligned}
$$

To improve the quality of the generalized Wannier functions, we minimize their total quadratic spread, i.e, the sum of their variances, ${ }^{24}$ which is given by

$$
\Omega=\sum_{j=1}^{J} \tilde{\sigma}_{j}^{2}=\overline{\operatorname{Tr}[\tilde{Y}(k)]}-\sum_{j=1}^{J} \tilde{x}_{j}^{2} .
$$

Since similar matrices have the same trace, this functional takes the form

$$
\begin{aligned}
\Omega & =\overline{\operatorname{Tr}\left[\mathbb{Y}(k)-\mathbb{X}^{2}(k)+\tilde{\mathbb{X}}^{\dagger}(k) \tilde{\mathbb{X}}(k)\right]}-\sum_{j=1}^{J}\left[\overline{\tilde{X}_{j, j}(k)}\right]^{2} \\
& =\Omega_{0}+2 \sum_{j=1}^{J} \sum_{j^{\prime}=1}^{j-1} \overline{\left|\tilde{X}_{j, j^{\prime}}\right|^{2}}+\sum_{j=1}^{J}\left[\overline{\tilde{X}_{j, j}^{2}}-\left(\overline{\tilde{X}_{j, j}}\right)^{2}\right],
\end{aligned}
$$

where $\Omega_{0}=\overline{\operatorname{Tr}\left[\mathbb{Y}-\mathbb{X}^{2}\right]}$ does not depend on $\mathbb{U}$. The second and third terms in the second line of Eq. (12) are non-negative. Therefore, $\Omega_{0}$ is a lower bound for the total spread. Moreover, if $\Omega_{0}$ is the minimum, then $\tilde{\mathbb{X}}(k)$ should be a real and constant diagonal matrix. According to Eq. (8), the optimization problem reduces to finding diagonal elements of $\tilde{\mathbb{X}}$ such that a unitary transformation having the periodicity of the reciprocal lattice solves the first-order differential equation

$$
\dot{U}(k)=i[\mathbb{X}(k) \mathbb{U}(k)-\mathbb{U}(k) \tilde{\mathbb{X}}] .
$$

The resulting GWFs are real if the solution satisfies

$$
\mathbb{U}(-k)=\mathbb{U}^{*}(k) .
$$

We note that expressions in Eq. (12) resemble the previous approach in Ref. 24, though we limit ourselves to the particular case of a 1D crystal. After some algebraic work, it is possible to prove that Eqs. (14), (19), and (20) in Ref. 24 correspond to the first, second, and third terms, respectively, in the second line of the present Eq. (12). In this latter version, however, only matrix elements of finite matrices $\mathbb{Y}(k), \mathbb{X}(k)$, and $\tilde{\mathbb{X}}(k)$ are involved, the invariance of the first term is more apparent, and the minimum variance is simply given in terms of $\mathbb{Y}(k)$ and $\mathbb{X}(k)$. Moreover, for the case of a general 1D crystal, it has been shown that eigenfunctions of the band-projected position operator are maximally localized GWFs. ${ }^{24}$ Therefore, diagonalization of that operator would lead to an optimal matrix $\mathbb{U}$. Instead, in next section, such a matrix is obtained by solving Eq. (13), and analytic solutions are given for two consecutive bands in a $1 \mathrm{D}$ crystal having inversion symmetry. Later on, such solutions are compared with those of the eigenvalue problem for the band-projected position operator. 


\section{EQUATIONS FOR TWO BANDS}

Now we limit ourselves to the case of two bands, i.e., $J=2$. Since $\mathbb{U}(k)$ is unitary, we have $\operatorname{det}[\mathbb{U}(k)]=\exp [i \tau(k)]$, where $\tau(k)$ is a real function. Moreover, we obtain $U_{1,2}=-U_{2,1}^{*} \exp (i \tau), U_{2,2}=U_{1,1}^{*} \exp (i \tau)$, and $|\operatorname{det}[\mathbb{U}]|=$ $\left|U_{1,1}\right|^{2}+\left|U_{2,1}\right|^{2}=1$. Thus, there exist real functions $\theta(k)$, $\phi_{1,1}(k)$, and $\beta(k)$, such that $U_{1,1}=\cos (\theta) \exp \left(i \phi_{1,1}\right)$ and $U_{2,1}=\sin (\theta) \exp \left[i\left(\phi_{1,1}+\beta\right)\right]$. Since $\left|U_{2,2}\right|=\left|U_{1,1}\right|$, we conveniently denote the phase difference between $U_{2,2}$ and $U_{1,1}$ as $\alpha(k)$. Hence, matrix $\mathbb{U}$ may be written as

$$
\mathbb{U}=e^{\frac{i \tau}{2}}\left(\begin{array}{lc}
\cos (\theta) & -\sin (\theta) e^{-i \beta} \\
\sin (\theta) e^{i \beta} & \cos (\theta)
\end{array}\right)\left(\begin{array}{cc}
e^{-\frac{i \alpha}{2}} & 0 \\
0 & e^{\frac{i \alpha}{2}}
\end{array}\right) .
$$

We assume that the WFs of each single band have been already optimized. Consequently, ${ }^{23} X_{1,1}=x_{1}$ and $X_{2,2}=x_{2}$. Also, since $\mathbb{X}$ is Hermitian, diagonal terms are real, while offdiagonal ones satisfy $X_{1,2}=X_{2,1}^{*}$, with $X_{2,1}=C \exp (i \gamma)$, where $C(k)$ and $\gamma(k)$ are real functions. Hence, assuming $\mathbb{U}(k)$ leads to a global minimum $\Omega_{0}$, we have $\tilde{X}_{1,1}=\tilde{x}_{1}, \tilde{X}_{2,2}=\tilde{x}_{2}$, and $\tilde{X}_{1,2}=\tilde{X}_{2,1}=0$. In this way, the sum of variances of the maximally localized GWFs is

$$
\tilde{\sigma}_{1}^{2}+\tilde{\sigma}_{2}^{2}=\Omega_{0}=\sigma_{1}^{2}+\sigma_{2}^{2}-2 \overline{C^{2}} .
$$

Moreover, Eq. (13) leads to a rather simple system of differential equations, namely,

$$
\begin{gathered}
\dot{\tau}=\left(x_{1}+x_{2}\right)-\left(\tilde{x}_{1}+\tilde{x}_{2}\right), \\
\dot{\theta}=C \sin (\beta-\gamma), \\
\dot{\alpha}=\Delta x-\Delta \tilde{x}-2 C \cos (\beta-\gamma) \tan (\theta),
\end{gathered}
$$

and

$$
\dot{\beta}=\Delta x+2 C \cos (\beta-\gamma) \cot (2 \theta),
$$

where $\Delta x=x_{2}-x_{1}$ and

$$
\begin{aligned}
\Delta \tilde{x}= & \tilde{x}_{2}-\tilde{x}_{1} \\
= & \Delta x-2 \overline{C \cos (\beta-\gamma) \tan (\theta)} \\
& -\frac{a}{2 \pi}[\alpha(\pi / a)-\alpha(-\pi / a)] .
\end{aligned}
$$

The periodicity of $\mathbb{U}$ leads to conditions $\tau(k+$ $2 \pi / a)=\tau(k)+2 \pi \mu_{\tau}, \quad \theta(k+2 \pi / a)=(-1)^{\mu_{\beta}} \theta(k)+\pi \mu_{\theta}$, $\alpha(k+2 \pi / a)=\alpha(k)+2 \pi \mu_{\alpha}$, and $\beta(k+2 \pi / a)=\beta(k)+$ $\pi \mu_{\beta}$, where $\mu_{\tau}, \mu_{\theta}, \mu_{\alpha}$, and $\mu_{\beta}$ are integers, with $\mu_{\alpha}-$ $\mu_{\tau}$ and $\mu_{\theta}$ having the same parity. Moreover, Eq. (14) implies $\tau(-k)=-\tau(k)+2 \pi v_{\tau}, \theta(-k)=(-1)^{\nu_{\beta}} \theta(k)+\pi v_{\theta}$, $\alpha(-k)=-\alpha(k)+2 \pi v_{\alpha}$, and $\beta(-k)=-\beta(k)+\pi v_{\beta}$, where $v_{\tau}, v_{\theta}, v_{\alpha}$, and $v_{\beta}$ are integers, with $v_{\alpha}-v_{\tau}$ and $v_{\theta}$ having the same parity.

At this point we note that the expression in Eq. (15) for transformation matrix $\mathbb{U}$ has the advantage of the differential equation for $\tau(k)$ being decoupled. Solving Eq. (17) with the corresponding boundary conditions leads to $\tau(k)=a \mu_{\tau} k+$ $\pi v_{\tau}$, with $\mu_{\tau}=\left[\left(x_{1}+x_{2}\right)-\left(\tilde{x}_{1}+\tilde{x}_{2}\right)\right] / a$. Then, by taking $\mu_{\tau}=0$, the condition $\tilde{x}_{1}+\tilde{x}_{2}=x_{1}+x_{2}$ holds; i.e., the sum of centers of Wannier functions is conserved. ${ }^{24}$ Additionally, by choosing $\nu_{\tau}=0, \tau(k)$ identically vanishes. In other words, the matrix $\mathbb{U}$ belongs to the special unitary group $S U(2)$. Of course, in agreement with these choices, $\mu_{\alpha}$ and $\mu_{\theta}$ should have equal parities. The same thing applies to $v_{\alpha}$ and $v_{\theta}$.

Now we further restrict the discussion to the case of a 1D crystal with inversion symmetry and two consecutive bands labeled by $j=1$ and $j=2$. As shown in Appendix A, we may take $\gamma(k)=k \Delta x+s \pi / 2$, where $s=0(s=1)$ when the parities of $w_{1}(x)$ and $w_{2}(x)$ are different (equal). Moreover, $C(k)$ is even (odd) when $s=0(s=1)$, and $C(k)$ is periodic (antiperiodic) over the reciprocal lattice, when $n=2 \Delta x / a$ is an even (odd) integer. At this point we note that Bloch functions at the edges of a band gap have different parities. ${ }^{30}$ Therefore, the case $s=1$ and even $n$ does not occur for consecutive bands.

Since $\dot{\gamma}=\Delta x$, Eqs. (18)-(20) may be rewritten as

$$
\begin{gathered}
\dot{\theta}=C \sin (\xi), \\
\dot{\alpha}=2 \overline{C \cos (\xi) \tan (\theta)}-2 C \cos (\xi) \tan (\theta)+a \mu_{\alpha},
\end{gathered}
$$

and

$$
\dot{\xi}=2 C \cos (\xi) \cot (2 \theta),
$$

respectively, where $\xi=\beta-\gamma$. By combining Eqs. (22) and (24), it is easily shown that $\cos (\xi) \sin (2 \theta)=A$, where $A$ is a constant satisfying $-1 \leqslant A \leqslant 1$. In what follows, only cases $A=-1$ and $A=0$ need to be considered.

On the one hand, $A=-1$ solves the problem provided $\theta(k)=-\pi / 4, \dot{\alpha}=2(C-\bar{C})+a \mu_{\alpha}, \xi(k)=0$ [i.e., $\beta(k)=\gamma(k)], \mu_{\theta}=\left[(-1)^{n}-1\right] / 4, \mu_{\beta}=n=2 \Delta x / a, v_{\theta}=$ $\left[(-1)^{s}-1\right] / 4$, and $v_{\beta}=s$. Therefore, this solution applies for $s=0$ and even $n$ only, with $\mu_{\theta}=v_{\theta}=v_{\beta}=0$. Moreover, taking $\mu_{\alpha}=v_{\alpha}=0$, with the same parity as $\mu_{\theta}$ and $v_{\theta}$, Eq. (23) is solved to obtain

$$
\alpha(k)=2 \int_{0}^{k}[C(\kappa)-\bar{C}] d \kappa,
$$

where $\bar{C}$ is the mean value of $C(k)$. Furthermore, $\Delta \tilde{x}=$ $\Delta x+2 \bar{C}$ leads to $\tilde{x}_{1}=x_{1}-\bar{C}$ and $\tilde{x}_{2}=x_{2}+\bar{C}$. The optimal transformation matrix is

$$
\mathbb{U}=\frac{1}{\sqrt{2}}\left(\begin{array}{lc}
1 & e^{-i n k a / 2} \\
-e^{i n k a / 2} & 1
\end{array}\right)\left(\begin{array}{cc}
e^{-\frac{i \alpha}{2}} & 0 \\
0 & e^{\frac{i \alpha}{2}}
\end{array}\right) .
$$

On the other hand, $A=0$ solves the problem for odd values of $n=2 \Delta x / a$, if $\dot{\theta}=-C, \mu_{\theta}=v_{\theta}=0, \dot{\alpha}=a \mu_{\alpha}$, and $\xi(k)=-\pi / 2$, i.e., $\beta(k)=\gamma(k)-\pi / 2$, with $\mu_{\beta}=n$ and $v_{\beta}=s-1$. Since $\mu_{\alpha}$ and $v_{\alpha}$ should be even, we take $\mu_{\alpha}=$ $v_{\alpha}=0$, which leads to $\alpha(k)=0$ for every $k$. Moreover,

$$
\theta(k)=\int_{k}^{s \pi / a} C(\kappa) d \kappa
$$

is odd (even) for $s=0(s=1)$, and the difference between centers is preserved, i.e., $\Delta \tilde{x}=\Delta x$. Since the same happens with their sum, both centers remain untouched $\left(\tilde{x}_{1}=x_{1}\right.$ and $\tilde{x}_{2}=x_{2}$ ). The transformation matrix becomes

$$
\mathbb{U}=\left(\begin{array}{lc}
\cos (\theta) & -i^{1-s} \sin (\theta) e^{-i n k a / 2} \\
i^{s-1} \sin (\theta) e^{i n k a / 2} & \cos (\theta)
\end{array}\right) .
$$

It is worth noticing that we have obtained the forms of matrix $\mathbb{U}(k)$ leading to a constant, real diagonal matrix $\tilde{\mathbb{X}}(k)$, 
for two consecutive bands, in the three symmetry cases of interest. This guarantees $\tilde{\Omega}$ reaches its global minimum, which is given by Eq. (16).

\section{NUMERICAL RESULTS AND DISCUSSION}

To be concrete, we deal with a diatomic version of the Kronig-Penney model, ${ }^{31,32}$ where a particle of mass $m$ has potential energy given by

$$
V(x)=-\frac{v \hbar^{2}}{m a} \sum_{n}[\delta(x-n a+b / 2)+\delta(x-n a-b / 2)] .
$$

The index $n$ runs from $-\infty$ to $+\infty, v>0$ is the dimensionless interaction strength, and $b<a$ is an interatomic distance. The energy bands are obtained from $E=\hbar^{2} \varepsilon /\left(2 m a^{2}\right)$ and $\cos (k a)=\mu(\varepsilon)$, where

$$
\begin{aligned}
\mu(\varepsilon)= & \left(1-v^{2} / \varepsilon\right) \cos (\sqrt{\varepsilon})-2 v \sin (\sqrt{\varepsilon}) / \sqrt{\varepsilon} \\
& +v^{2} \cos [(1-2 b / a) \sqrt{\varepsilon}] / \varepsilon .
\end{aligned}
$$

Since the potential presents inversion centers at integer multiples of $a / 2$, phases of Bloch functions are chosen appropriately in order to obtain maximally localized Wannier functions for each isolated band. ${ }^{30}$ The numerical results below correspond to parameters $v=4$ and $b=3 a / 8$. Moreover, energies and Bloch functions are calculated over a sample of 400 wave vectors uniformly spaced in the first Brillouin zone.

Figures 1(a) and 1(b) display the WFs of the lower two bands. Hence, the functions are labeled by superscript $(1,2)$. It is apparent that single-band Wannier functions resemble molecular orbitals, with the first (second) band corresponding to the bonding (antibonding) one. Both molecular orbitals are centered at the origin of coordinates, i.e., $x_{1}=x_{2}=0$, and the first (second) one is even (odd), corresponding to the case $s=0$ and $n=0$ in Sec. III. Moreover, the standard deviation of the WF for the first (second) band is $\sigma_{1} \approx 0.2579 a$ $\left(\sigma_{2} \approx 0.3323 a\right)$. These functions decay exponentially with coefficients that may be given in terms of the quantities
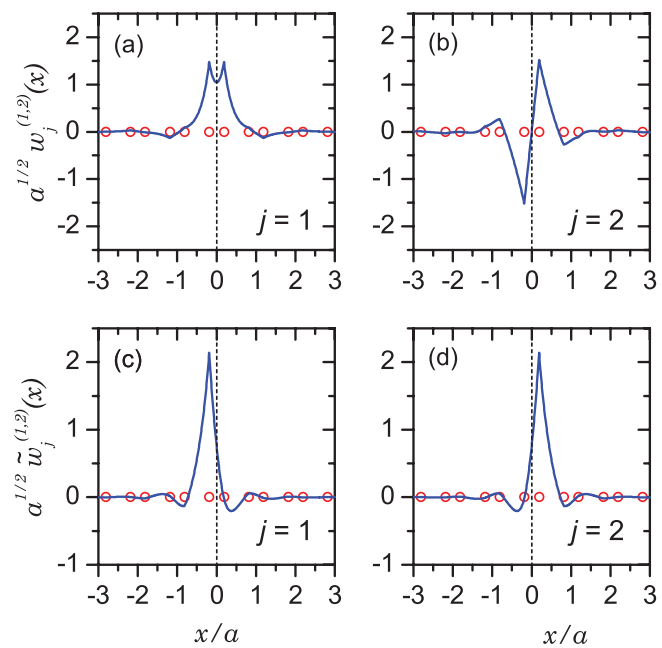

FIG. 1. (Color online) Wannier functions of (a) first and (b) second bands and (c,d) generalized Wannier functions for the pair $(1,2)$ of bands. Open circles indicate atomic positions. $h_{j}=a^{-1} \arccos \left[\mu\left(\varepsilon_{j}\right)\right]$, where $\varepsilon_{j}$ is the $j$ th root of $\mu^{\prime}(\varepsilon)=0$. For parameters in the figure, we have $h_{1} \approx 0.9969 a^{-1}$ and $h_{2} \approx 1.3542 a^{-1}$. Hence, ${ }^{21}$ the decay of the WF of the first (second) band is given by $h_{1}$ (the minimum between $h_{1}$ and $h_{2}$, i.e., $\left.h_{1}\right)$. This corresponds to the distance from the real $k$ axis to the closest branch points of $\varepsilon_{j}(k)$. Of course, an additional power-law decay with exponent $3 / 4$ also occurs. ${ }^{22}$

Generalized Wannier functions for the lower two bands are calculated by the transformation matrix in Eq. (26). Since $n=0$, it takes a simpler form, namely,

$$
\mathbb{U}(k)=\frac{1}{\sqrt{2}}\left(\begin{array}{ll}
e^{-\frac{i \alpha(k)}{2}} & e^{\frac{i \alpha(k)}{2}} \\
-e^{-\frac{i \alpha(k)}{2}} & e^{\frac{i \alpha(k)}{2}}
\end{array}\right),
$$

where $\alpha(k)$, given by Eq. (25), is an odd function. Figures 1(c) and 1(d) display results for the same parameters as Figs. 1(a) and $1(\mathrm{~b})$. The centers of these functions are $\tilde{x}_{1} \approx-0.21125 a$ and $\tilde{x}_{2} \approx 0.21125 a$. Moreover, they present identical standard deviations, namely $\tilde{\sigma}_{1}=\tilde{\sigma}_{2} \approx 0.1516 a$. This means that the total variance has decreased by about $74 \%$. It is worth noting that these GWFs are neither symmetric nor antisymmetric. Indeed, crystal symmetry manifests in the sense that they are mirror images of each other about line $x=0$, i.e., $\tilde{w}_{2}^{(1,2)}(x)=$ $\tilde{w}_{1}^{(1,2)}(-x)$. As shown in Appendix B, this follows from choices leading to Eq. (31) and gauge choices ${ }^{21} \psi_{1, k}(-x)=\psi_{1,-k}(x)$ and $\psi_{2, k}(-x)=-\psi_{2,-k}(x)$. We also note that, because of the diagonal character of $\tilde{\mathbb{X}}(k)$, the condition $\left\langle\tilde{w}_{1}^{(1,2)}|x| \tilde{w}_{2}^{(1,2)}\right\rangle=0$ should apply. The symmetry relation between the GWFs also leads to this result, namely,

$$
\begin{aligned}
\left\langle\tilde{w}_{1}^{(1,2)}|x| \tilde{w}_{2}^{(1,2)}\right\rangle & =\int_{-\infty}^{\infty} x \tilde{w}_{1}^{(1,2)}(x) \tilde{w}_{2}^{(1,2)}(x) d x \\
& =\int_{-\infty}^{\infty} x \tilde{w}_{1}^{(1,2)}(x) \tilde{w}_{1}^{(1,2)}(-x) d x=0,
\end{aligned}
$$

since $\tilde{w}_{1}^{(1,2)}(x) \tilde{w}_{1}^{(1,2)}(-x)$ is an even function.

Regarding the asymptotic behavior of GWFs, symmetry allows us to focus on $\tilde{w}_{1}^{(1,2)}(x)$ only. We notice that both the coefficient of exponential decay and the exponent of the power-law decay may be extracted from the list of probabilities for the particle to be found within each unit cell. ${ }^{22,23}$ For the functions in the figure, we have obtained identical parameters for $x \rightarrow-\infty$ and $x \rightarrow \infty$. Namely, the coefficient of exponential localization is noticeably larger for these generalized WFs than for functions in Figs. 1(a) and 1(b), and it is found to be $h_{2} \approx 1.3542$. This is due to cancellation between terms in the linear combination of $\psi_{1, k}$ and $\psi_{2, k}$ at their common branch points, while farther branch points of $\psi_{2, k}$ survive. The $3 / 4$ power-law decay also occurs.

When a minimization procedure of total variance is applied to the pair $(2,3)$ of bands, the results are quite different. This is because the symmetries of WFs of second and third bands are different, while the distance between their centers is an odd multiple of half period. In fact, it is apparent in Figs. 2(a) and 2(b) that $x_{1}=0$ and $x_{2}=a / 2$. Moreover, the variance and coefficient of exponential localization for the second (third) band are $\sigma_{1} \approx 0.3323 a$ and $h_{1}=0.9968 a^{-1}$ ( $\sigma_{2} \approx 0.3468 a$ and $h_{3}=0.7994 a^{-1}$ ), respectively. This case is characterized by parameters $s=1$ and $n=1$ of Sec. III. Hence, the transformation in Eq. (28) is used to obtain 

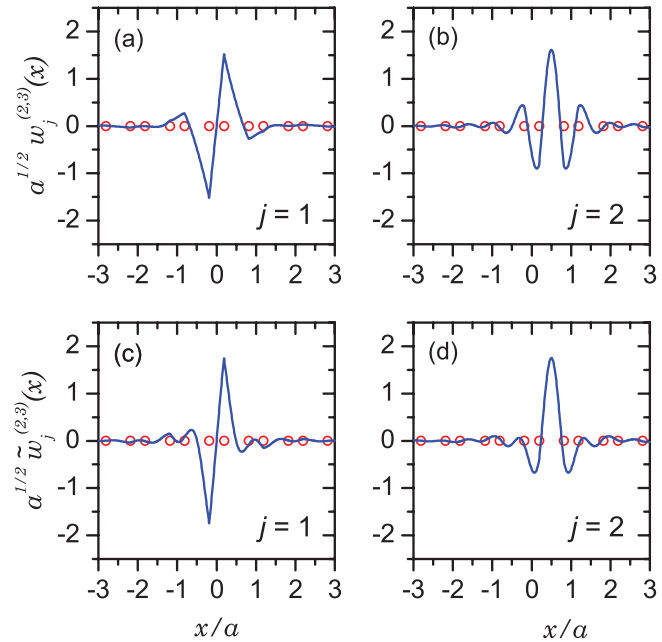

FIG. 2. (Color online) Wannier functions of (a) second and (b) third bands and (c,d) generalized Wannier functions for the pair $(2,3)$ of bands. Open circles indicate atomic positions.

the generalized functions shown in Figs. 2(c) and 2(d). By comparing WFs and GWFs, we observe that the centers and symmetries are preserved, while the total spread diminishes. On the one hand, the properties $\tilde{w}_{1}^{(2,3)}(-x)=-\tilde{w}_{1}^{(2,3)}(x)$ and $\tilde{w}_{2}^{(2,3)}(a-x)=\tilde{w}_{2}^{(2,3)}(x)$ are shown in Appendix B to follow from Eq. (28) and gauge choices ${ }^{21} \psi_{2, k}(-x)=-\psi_{2,-k}(x)$ and $\psi_{3, k}(a-x)=\psi_{3,-k}(x)$. On the other hand, the variances of $w_{1}^{(2,3)}(x)$ and $w_{2}^{(2,3)}(x)$ are $\tilde{\sigma}_{1} \approx 0.3157 a$ and $\tilde{\sigma}_{2} \approx 0.2108 a$, respectively, corresponding to a decrease in total variance of about $37 \%$. Furthermore, both GWFs decay exponentially with the same coefficient, namely, $h_{3}=0.7994 a^{-1}$. To understand this, it may be noticed that the first, second, and third gaps with coefficients $h_{1}, h_{2}$, and $h_{3}$ are relevant for the pair $(2,3)$ of bands. Therefore, even if cancellation at branch points of the second gap were to occur, $\min \left(h_{1}, h_{3}\right)=h_{3}$ would dominate the decay.

The results are similar for the pair $(3,4)$ of bands. As shown in Figs. 3(a) and 3(b), now single-band WFs have
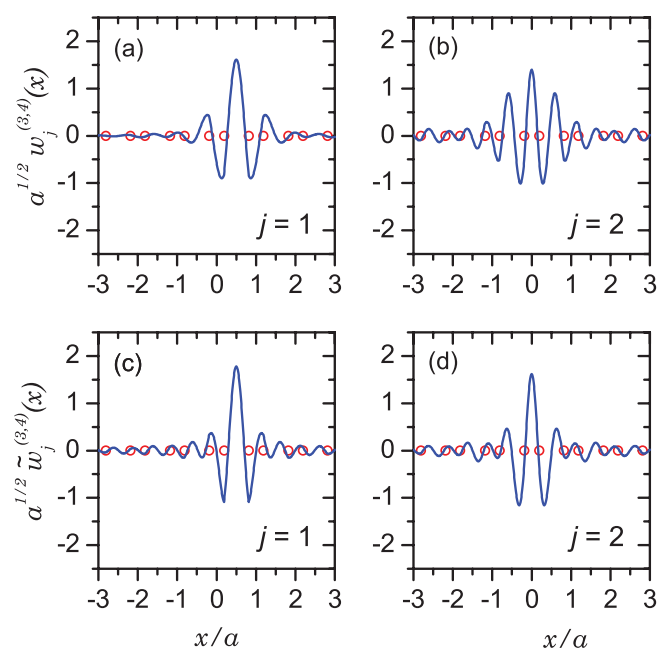

FIG. 3. (Color online) Wannier functions of (a) third and (b) fourth bands and (c,d) generalized Wannier functions for the pair $(3,4)$ of bands. Open circles indicate atomic positions. the same parity but their centers are separated by half a period. Therefore, the transformation in Eq. (28) leads to the maximally localized GWFs displayed in Figs. 3(c) and 3(d). Clearly, centers and symmetries are preserved again. Namely, the conditions $\tilde{w}_{1}^{(3,4)}(a-x)=\tilde{w}_{1}^{(3,4)}(x)$ and $\tilde{w}_{2}^{(3,4)}(-x)=\tilde{w}_{2}^{(3,4)}(x)$ hold. These properties may be derived from Eq. (28) and gauge choices ${ }^{21} \psi_{3, k}(a-x)=\psi_{3,-k}(x)$ and $\psi_{4, k}(-x)=\psi_{4,-k}(x)$ (see Appendix B). Moreover, standard deviations of WFs $\left(\sigma_{1}=0.3468 a, \sigma_{2}=1.1982 a\right)$ and GWFs $\left(\tilde{\sigma}_{1}=0.6915 a, \tilde{\sigma}_{2}=0.9618 a\right)$, corresponding to a decrease in total spread of about $10 \%$. It is interesting to note in Fig. 3 that one function has become broader [Fig. 3(a) $\rightarrow$ Fig. 3(c)] while the other has narrowed [Fig. 3(b) $\rightarrow$ Fig. 3(d)]. At the same time, the first one has slowed down its exponential decrease. In fact, the coefficient of decay of both GWFs is found to be $h_{4} \approx 0.0545 a^{-1}$.

For the sake of completeness, we now compare the results presented above with those obtained by diagonalization of the position operator $x$. Conveniently, this operator can be represented in terms of Wannier functions $w_{j, n}(x)$, with $j=$ $1, \ldots, J$ and $n$ running over the integers. The matrix elements of $x$ in this basis are given by

$$
x_{(j, n),\left(j^{\prime}, n^{\prime}\right)}=\left\langle w_{j, n}|x| w_{j^{\prime}, n^{\prime}}\right\rangle=X_{j, j^{\prime}, n^{\prime}-n}+n a \delta_{j, j^{\prime}} \delta_{n, n^{\prime}},
$$

with

$$
X_{j, j^{\prime}, n}=\overline{\exp \left(- \text { inak) } X_{j, j^{\prime}}(k)\right.}
$$

being the $n$th Fourier coefficient of $X_{j, j^{\prime}}(k)$. Clearly, the matrix is Hermitian, and its eigenvalues are the centers of GWFs with maximal localization. ${ }^{24}$ It is worth noting that the spectrum of $x$ is periodic, with period $a$, and the corresponding eigenvectors are the coordinates of GWFs in the basis set of single-band WFs. Therefore, it suffices to accurately determine those eigenvalues between $-a / 2$ and $a / 2$. With this in mind, approximate eigenvalues may be calculated by truncating the basis, namely, by taking $n=-N, 1-N, \ldots,-1,0,1, \ldots, N$, for a sufficiently large integer $N$. The truncated matrix is of dimension $J(2 N+1) \times$ $J(2 N+1)$, and accuracy may be improved by increasing the value of $N$. This is apparent in Fig. 4, where the two relevant eigenvalues for the pair $(1,2)$ of bands are shown as a function of $N$. In fact, the lower [upper] eigenvalue in Fig. 4(a)
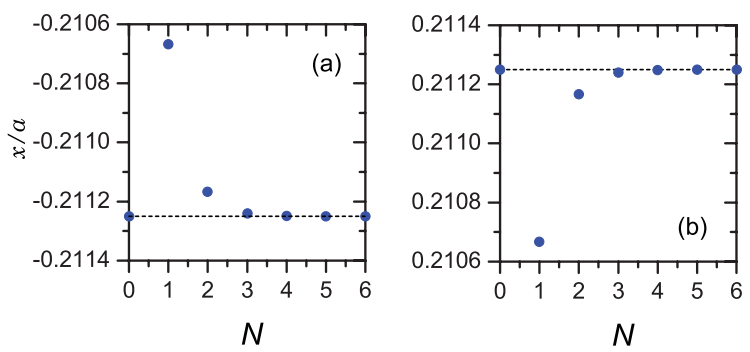

FIG. 4. (Color online) The two eigenvalues, within the range $-a / 2<x \leqslant a / 2$, of the position operator, when projected on the set of Wannier functions $w_{j, n}(x)$, with $j=1$ or 2 and $n=$ $-N, 1-N, \ldots, N$. Dots denote the lower (upper) eigenvalue, with $N=0,1, \ldots, 6$. Horizontal dashed lines are at the centers of the generalized Wannier functions in Fig. 1. 
[Fig. 4(b)] rapidly converges to the center of $\tilde{w}_{1}(x)\left[\tilde{w}_{2}(x)\right]$ as $N$ increases. It should also be remarked that eigenvalues for $N=0$ coincide with the corresponding limit values. This is in agreement with the analytical development in Sec. II, where $\tilde{x}_{1}$ and $\tilde{x}_{2}$ have been shown to be $x_{1}-\bar{C}$ and $x_{2}+\bar{C}$, respectively, with $x_{1}=x_{2}=0$ and $\bar{C}=x_{(1,0),(2,0)}=x_{(2,0),(1,0)}$. We have also checked the convergence to GWFs of linear combinations of the $J(2 N+1)$ WFs, with the coefficients being the corresponding eigenvectors. For the considered parameters of the $1 \mathrm{D}$ crystal, the scalar product between the analytically obtained GWFs and the linear combinations is above 0.99 , for all values of $N$. And, apparently, convergence to 1 is quite rapid. Nevertheless, good agreement between the exponentially decaying tails may require large values of $N$. A similar analysis may be done for other pairs of bands.

\section{CONCLUSIONS}

The total variance of generalized Wannier functions of two consecutive bands in a $1 \mathrm{D}$ crystal with inversion symmetry has been minimized by following a straightforward analytical approach. This has led to real generalized Wannier functions of maximal localization. Different expressions have been given for the cases where the distance between the centers of single-band Wannier functions is either an even or an odd multiple of half a period of the crystal. Numerical results were presented for the lowest four bands of a particle subject to a diatomic Kronig-Penney potential. For the lowest couple of bands, calculated single-band (generalized) Wannier functions resemble molecular (orthogonalized atomic) orbitals, and the latter display increased exponential localization. Moreover, the centers change and the generalized functions are neither symmetric nor antisymmetric, but they are related by an inversion-symmetry transformation. Instead, for the second and third pairs of consecutive bands, Wannier functions conserve both center and inversion symmetry, while exponential decay does not increase. Changes in the coefficient of such decay have been explained in terms of branch points of Bloch functions of each band.

The presented explicit expressions of the matrix that transforms the Bloch function into quasi-Bloch functions noticeably simplify computation of generalized Wannier functions, making them available to a broader community. Moreover, convergence of truncation and iterative procedures may be further tested. This has been done for the eigenvalue problem of the band-projected position operator. At the same time, interesting properties of symmetry and decay have been explained. Calculated functions should find applications in the study of defect-induced localized states with energy lying within the gap separating the considered pair of bands, but future work should deal with generalized Wannier functions of several bands. We expect similar efforts will be devoted to one-dimensional crystals lacking inversion symmetry, as well as two- and three-dimensional crystals.

\section{ACKNOWLEDGMENTS}

We thank the Brazilian Agencies CNPq and FAPESP for financial support.

\section{APPENDIX A: OFF-DIAGONAL TERM $X_{2,1}(k)$}

In this section, properties of real functions $C(k)$ and $\gamma(k)$ satisfying $X_{2,1}(k)=C(k) \exp [i \gamma(k)]$ are demonstrated. From Eq. (3), we have

$$
\begin{aligned}
X_{2,1}(k) & =i \int_{0}^{a} u_{2, k}^{*}(x) \dot{u}_{1, k}(x) d x \\
& =\int_{0}^{a} \psi_{2, k}^{*}(x)\left[i \dot{\psi}_{1, k}(x)+x \psi_{1, k}(x)\right] d x .
\end{aligned}
$$

If $w_{j}(x)$ obeys $w_{j}(x)=s_{j} w_{j}\left(2 x_{j}-x\right)$, then it is even (odd) about $x=x_{j}$, when $s_{j}=1 \quad\left(s_{j}=-1\right)$. The corresponding Bloch function satisfies $\psi_{j, k}(x)=s_{j} \psi_{j,-k}\left(2 x_{j}-x\right)=$ $s_{j} \psi_{j, k}^{*}\left(2 x_{j}-x\right)$. Taking the Bloch condition and orthogonality of Bloch functions into account, we obtain

$$
\begin{aligned}
X_{2,1}(k)= & s_{1} s_{2} \int_{2 x_{1}-a}^{2 x_{1}} \psi_{2, k}\left(2 x_{2}-x\right)\left[i \dot{\psi}_{1, k}^{*}\left(2 x_{1}-x\right)\right. \\
& \left.+x \psi_{1, k}^{*}\left(2 x_{1}-x\right)\right] d x \\
= & s_{1} s_{2} e^{2 i k \Delta x} \int_{0}^{a} \psi_{2, k}(x)\left[i \dot{\psi}_{1, k}^{*}(x)\right. \\
& \left.+\left(2 x_{1}-x\right) \psi_{1, k}^{*}(x)\right] d x \\
= & -s_{1} s_{2} e^{2 i k \Delta x} X_{2,1}^{*}(k) .
\end{aligned}
$$

Therefore, we may write

$$
e^{-i k \Delta x} X_{2,1}(k)=(-1)^{s}\left[e^{-i k \Delta x} X_{2,1}(k)\right]^{*},
$$

where $(-1)^{s}=-s_{1} s_{2}$, and we take $s=0(s=1)$ when the parities of $w_{1}(x)$ and $w_{2}(x)$ are different (coincide). In the first (second) case, $e^{-i k \Delta x} X_{2,1}(k)$ is real [pure imaginary] and may be written as $C(k)[i C(k)]$. These results may be summarized as $X_{2,1}(k)=C(k) \exp [i \gamma(k)]$, where $C(k)$ is real and $\gamma(k)=k \Delta x+s \pi / 2$.

From Eq. (A1) we may derive inversion-symmetry properties of $C(k)$. In fact, since $X_{2,1}(-k)=X_{2,1}^{*}(k)$, we obtain $C(-k)=(-1)^{s} C(k)$. This means $C(k)$ is even (odd) for $s=0$ $(s=1)$. Furthermore, since $X_{2,1}(k)$ has the periodicity of the reciprocal lattice, $C(k)$ satisfies $C(k+2 \pi / a)=(-1)^{n} C(k)$, with $n=2 \Delta x / a$; i.e., it is periodic (antiperiodic) when $n$ is even (odd).

\section{APPENDIX B: SYMMETRY AND GENERALIZED WANNIER FUNCTIONS}

To obtain a symmetry relation between the generalized Wannier functions of a couple of bands having Wannier functions centered at $x=0$ with the first (second) one being an even (odd) function, we take into account conditions $\psi_{1, k}(-x)=$ $\psi_{1,-k}(x)$ and $\psi_{2, k}(-x)=-\psi_{2,-k}(x)$. From Eq. (31), we have

$$
\tilde{\psi}_{1, k}^{(1,2)}(x)=\frac{e^{-\frac{i \alpha(k)}{2}}}{\sqrt{2}}\left[\psi_{1, k}(x)-\psi_{2, k}(x)\right]
$$

and

$$
\tilde{\psi}_{2, k}^{(1,2)}(x)=\frac{e^{\frac{i \alpha(k)}{2}}}{\sqrt{2}}\left[\psi_{1, k}(x)+\psi_{2, k}(x)\right] .
$$


Moreover, according to Eq. (25), $\alpha(k)$ is an odd function, and we obtain

$$
\tilde{\psi}_{2, k}^{(1,2)}(x)=\frac{e^{\frac{-i \alpha(-k)}{2}}}{\sqrt{2}}\left[\psi_{1,-k}(-x)-\psi_{2,-k}(-x)\right]=\tilde{\psi}_{1,-k}^{(1,2)}(-x) .
$$

Taking the average over the first Brillouin zone, this leads to $\tilde{w}_{2}^{(1,2)}(x)=\tilde{w}_{1}^{(1,2)}(-x)$.

Now, let us try to demonstrate that centers and symmetries of $\tilde{w}_{1}(x)$ and $\tilde{w}_{2}(x)$ coincide with those of $w_{1}(x)$ and $w_{2}(x)$, respectively, when $n=2 \Delta x / a$ is odd. To be concrete, we consider the pair $(2,3)$ in Sec. IV, where $\psi_{2, k}(-x)=-\psi_{2,-k}(x)$ and $\psi_{3, k}(a-x)=\psi_{3,-k}(x)$. We note that $s=0$ (opposite symmetries), $\theta(k)$ is odd, and $n=1$. Then, from Eq. (28), we have

$$
\tilde{\psi}_{1, k}^{(2,3)}(x)=\cos (\theta(k)) \psi_{2, k}(x)-i e^{\frac{i k a}{2}} \sin (\theta(k)) \psi_{3, k}(x)
$$

and

$$
\tilde{\psi}_{2, k}^{(2,3)}(x)=-i e^{-\frac{i k a}{2}} \sin (\theta(k)) \psi_{2, k}(x)+\cos (\theta(k)) \psi_{3, k}(x) .
$$

According to the Bloch condition, this leads to

$$
\begin{aligned}
\tilde{\psi}_{1, k}^{(2,3)}(-x)= & -\cos (-\theta(-k)) \psi_{2,-k}(x) \\
& -i e^{\frac{-i k a}{2}} \sin (-\theta(-k)) \psi_{3,-k}(x) \\
= & -\cos (\theta(-k)) \psi_{2,-k}(x) \\
& +i e^{\frac{-i k a}{2}} \sin (\theta(-k)) \psi_{3,-k}(x) \\
= & -\tilde{\psi}_{1,-k}^{(2,3)}(x)
\end{aligned}
$$

and

$$
\begin{aligned}
\tilde{\psi}_{2, k}^{(2,3)}(a-x)= & i e^{\frac{i k a}{2}} \sin (-\theta(-k)) \psi_{2,-k}(x) \\
& +\cos (-\theta(-k)) \psi_{3,-k}(x) \\
= & -i e^{\frac{i k a}{2}} \sin (\theta(-k)) \psi_{2,-k}(x) \\
& +\cos (\theta(-k)) \psi_{3,-k}(x) \\
= & \tilde{\psi}_{2,-k}^{(2,3)}(x) .
\end{aligned}
$$

Hence, after averaging over the first Brillouin zone, the results are $\tilde{w}_{1}^{(2,3)}(-x)=-\tilde{w}_{1}^{(2,3)}(x)$ and $\tilde{w}_{2}^{(2,3)}(a-x)=\tilde{w}_{2}^{(2,3)}(x)$.

The conservation of symmetry for pair $(3,4)$ in Sec. IV may be proved in the same way. In doing so, the conditions $\psi_{3, k}(a-$ $x)=\psi_{3,-k}(x)$ and $\psi_{4, k}(-x)=\psi_{4,-k}(x)$ should be taken into account. Moreover, we note that $s=1$ (same symmetry), $\theta(k)$ is even, and $n=-1$.
${ }^{1}$ R. Resta, Eur. Phys. J. B 79, 121 (2011).

${ }^{2}$ E. Krüger and H. Strunk, J. Supercond. Novel Mag. 24, 2103 (2011).

${ }^{3}$ T. Berlijn, D. Volja, and W. Ku, Phys. Rev. Lett. 106, 077005 (2011). ${ }^{4}$ T. Bazhirov, J. Noffsinger, and M. L. Cohen, Phys. Rev. B 84, 125122 (2011).

${ }^{5}$ T. Stollenwerk, R. Frank, A. Lubatsch, O. Zaitsev, S. Zhukovsky, D. Chigrin, and J. Kroha, Appl. Phys. B 105, 163 (2011).

${ }^{6}$ M. Lorenz, D. Usvyat, and M. Schütz, J. Chem. Phys. 134, 094101 (2011).

${ }^{7}$ D. Benson, O. F. Sankey, and U. Häussermann, Phys. Rev. B 84, 125211 (2011).

${ }^{8}$ J. S. Lee, G. A. H. Schober, M. S. Bahramy, H. Murakawa, Y. Onose, R. Arita, N. Nagaosa, and Y. Tokura, Phys. Rev. Lett. 107, 117401 (2011).

${ }^{9}$ M. Casula, M. Calandra, G. Profeta, and F. Mauri, Phys. Rev. Lett. 107, 137006 (2011).

${ }^{10}$ M. Shelley, N. Poilvert, A. A. Mostofi, and N. Marzari, Comput. Phys. Commun. 182, 2174 (2011).

${ }^{11}$ M. Chen, G.-C. Guo, and L. He, J. Phys.: Condens. Matter 23, 325501 (2011).

${ }^{12}$ N. W. Ashcroft and N. D. Mermin, Solid State Physics (Brooks Cole, Belmont, 1976).

${ }^{13} \mathrm{O}$. Madelung, Introduction to Solid-State Physics (Springer, Berlin, 1978).

${ }^{14}$ R. M. Martin, Basic Electronic Structure (Cambridge University Press, Cambridge, 2004).
${ }^{15}$ Conceptual Foundations of Materials, edited by S. G. Louie and M. L. Cohen (Elsevier, Amsterdam, 2006).

${ }^{16}$ Condensed Matter Physics, 2nd ed., edited by M. P. Marder (Wiley, Hoboken, 2010).

${ }^{17}$ G. H. Wannier, Phys. Rev. 52, 191 (1937).

${ }^{18}$ G. Nenciu, Commun. Math. Phys. 91, 81 (1983).

${ }^{19}$ C. Brouder, G. Panati, M. Calandra, C. Mourougane, and N. Marzari, Phys. Rev. Lett. 98, 046402 (2007).

${ }^{20}$ H. D. Cornean, A. Nenciu, and G. Nenciu, J. Phys. A 41, 125202 (2008).

${ }^{21}$ W. Kohn, Phys. Rev. 115, 809 (1959).

${ }^{22}$ L. He and D. Vanderbilt, Phys. Rev. Lett. 86, 5341 (2001).

${ }^{23}$ A. Bruno-Alfonso and D. R. Nacbar, Phys. Rev. B 75, 115428 (2007).

${ }^{24}$ N. Marzari and D. Vanderbilt, Phys. Rev. B 56, 12847 (1997).

${ }^{25}$ E. I. Blount, Solid State Phys. 13, 305 (1962).

${ }^{26}$ J. D. Cloizeaux, Phys. Rev. 135, A685 (1964); 135, A698 (1964).

${ }^{27}$ G. Eilenberger, Z. Phys. A 180, 43 (1964).

${ }^{28}$ A. A. Mostofi, J. R. Yates, Y.-S. Lee, I. Souza, D. Vanderbilt, and N. Marzari, Comput. Phys. Commun. 178, 685 (2008).

${ }^{29}$ J. Bhattacharjee and U. V. Waghmare, Phys. Rev. B 71, 045106 (2005).

${ }^{30}$ A. Bruno-Alfonso and G.-Q. Hai, J. Phys.: Condens. Matter 15, 6701 (2003).

${ }^{31}$ F. B. Pedersen, G. T. Einevoll, and P. C. Hemmer, Phys. Rev. B 44, 5470 (1991).

${ }^{32}$ M. J. Rave and W. C. Kerr, Eur. Phys. J. B 45, 473 (2005). 